\title{
Reevaluation of risk factors for time to subsequent events after first stroke occurrence using a new weighted all-cause effect measure
}

\section{Ann-Kathrin Ozga ( $\square$ a.ozga@uke.de )}

University Medical Center Hamburg-Eppendorf https://orcid.org/0000-0002-9501-7301

\section{Bernhard Rauch}

Stiftung Institut fur Herzinfarktforschung

\section{Frederick Palm}

HELIOS Klinikum Schwerin

\section{Christian Urbanek}

Department of Neurology, University of Heidelberg

\section{Armin Grau}

Department of Neurology, University of Heidelberg

\section{Heiko Becher}

Institute of Medical Biometry and Epidemiology, University Medical Center Hamburg-Eppendorf

\section{Geraldine Rauch}

Charite Universitatsmedizin Berlin

\section{Research article}

Keywords: stroke, death, composite endpoint, time-to-event, risk factors, weighted all-cause hazard ratio

Posted Date: April 24th, 2020

DOI: https://doi.org/10.21203/rs.2.19014/v3

License: (a) This work is licensed under a Creative Commons Attribution 4.0 International License.

Read Full License

Version of Record: A version of this preprint was published at BMC Public Health on June 1st, 2020. See the published version at https://doi.org/10.1186/s12889-020-08971-4. 


\section{Abstract}

Background: Risk diseases and risk factors for stroke include atrial fibrillation, hypertension, diabetes mellitus, smoking, and elevated LDL-cholesterol. Due to modern treatment options, the impact of these risk diseases on subsequent cardiovascular events or death after a first stroke is less clear and needs to be elucidated. We therefore aimed to get insights into the persistence of adverse prognostic effects of these risk diseases and risk factors on subsequent stroke or death events one year after the first stroke by using the new weighted all-cause hazard ratio.

Methods: This study evaluates the one year follow-up of 470 first ever stroke cases identified in the area of Ludwigshafen, Germany, with 23 deaths and 34 subsequent stroke events. For this purpose, the recently introduced "weighted all-cause hazard ratio" was used, which allows a weighting of the competing endpoints within a composite endpoint. Moreover, we extended this approach to allow an adjustment for covariates.

Results: None of these risk factors and risk diseases, most probably being treated after the first stroke, remained to be associated with a subsequent death or stroke [weighted hazard ratios ( $95 \%$ confidence interval) for diabetes mellitus, atrial fibrillation, high cholesterol, hypertension, and smoking are 0.4 (0.2 $0.9), 0.8(0.4-2.2), 1.3(0.5$ - 2.5), $1.2(0.3-2.7), 1.6$ (0.8 - 3.6), respectively]. However, when analyzed separately in terms of death and stroke, the risk factors and risk diseases under investigation affect the subsequent event rate to a variable degree.

Conclusions: Using the new weighted hazard ratio, established risk factors and risk diseases for the occurrence of a first stroke do not remain to be significant predictors for subsequent events like death or recurrent stroke. It has been demonstrated that the new weighted hazard ratio can be used for a more adequate analysis of cardiovascular risk and disease progress. The results have to be confirmed within a larger study with more events.

\section{Background}

Stroke is the second leading cause of death worldwide [1, 2]. Studies showed a risk of dying one year after a first stroke of around 40 percent $[3,4]$. Commonly known risk factors and risk diseases for a first ever stroke like atrial fibrillation, hypertension, diabetes mellitus, smoking, or high cholesterol are well accepted of being responsible for about 50-70 percent of all strokes [5, 6]. Risk factors for subsequent strokes after a first non-fatal stroke event were also analyzed but more heterogeneous results were gained with indicators including diabetes mellitus, coronary artery disease, hypertension, a high baseline score on the "National Institute of Health Stroke Scale", intracranial arterial stenosis, or hyperlipidemia [79]. Although a recent meta-analysis suggested that hypertension, diabetes mellitus, atrial fibrillation, and coronary heart disease might be risk factors for a subsequent stroke occurrence, they did not consider a time-to-event analysis[10]. Even less analyzed are risk factors for the "time to death" after a first ever stroke, which might differ from the common risk factors and risk diseases for the "time to first ever 
stroke". Although the rate of dying as a direct consequence of a stroke was reported to be high, it appeared to be difficult to quantify additional risk factors and risk diseases that influence the disease progress after a first stroke $[3,4]$. Heterogeneous groups of patients may be one reason for this observation, as different stroke etiologies markedly influence the risk of subsequent events. Moreover, the consequent implementation of secondary prevention strategies - especially protective medication markedly reduces the rate of subsequent clinical events [11]. Therefore, patients at risk in secondary prevention after a first non-fatal stroke represent a special selection and need to be newly defined in general, but also in all-day care. Finally, there are extended efforts in medical research to prevent subsequent strokes after a first stroke, thereby continuously changing risk patterns in secondary prevention [12].

In the present study, we aim to deliver a reevaluation of potential risk factors and risk diseases for premature death or stroke after a first stroke event. Taking into account that death acts as a competing event to a subsequent non-fatal stroke, the composite endpoint with the two components "time to subsequent death" or "time to subsequent stroke" was taken as primary endpoint. These two endpoints are clearly of different clinical relevance, as a fatal event always represents the worst outcome. This has to be addressed in the analysis of composite endpoints. However, common statistical methods for composite endpoints are usually based on the all-cause hazard ratio, which ignores the different impact of the components. To overcome this problem, we recently introduced the weighted all-cause hazard ratio to simplify interpretation of a composite endpoint with competing components of different clinical relevance $[13,14]$. In the present study, this new methodologic approach was extended for a situation to incorporate an adjustment for confounders. This allows to adequately model a complex time-to-event framework, in which two competing events with different clinical relevance are of primary interest.

The primary aim of this exploratory study was to (re)evaluate the effect of common risk factors and risk diseases for a first stroke on the "time to subsequent death or stroke" using a new weighted effect measure. As only 60 percent of patients survive more than one year after a first stroke, it is of high clinical relevance to estimate and characterize as exactly as possible the ongoing risk under actual treatment conditions $[3,4]$. We additionally aimed to illustrate how the weighted all-cause hazard ratio helps to more adequately describe the progress of risk along a chronic disease under contemporary treatment, a method of potential interest in public health.

\section{Methods}

\section{Study_population}

This is a study on $n=470$ incident cases with ischemic stroke with a 1-year follow-up. These cases are taken from a recently published case-control study [15] which was embedded in the Ludwigshafen population-based stroke registry covering about $93 \%$ of all stroke patients below the age of 80 years in the urbanized industrial area of Ludwigshafen [16]. Figure 1 shows the corresponding flow-chart. 
The diagnosis of "stroke" is based on the definition of the World Health Organization [17].

Cases gave written informed consent. They were of white ethnicity and between 18 and 80 years of age. Exclusion criteria were additional previous events like stroke of any etiology, acute transient ischemic attack, intracerebral, subdural or subarachnoidal hemorrhage, myocardial infarction within the previous 90 days, dementia, severe aphasia, and other relevant communication barriers as well as withdrawal of consent.

These cases, i.e. the underlying study population analyzed in this work, underwent a personal interview with trained interviewers using a standardized questionnaire. The interviewers were guided by a handbook. Instructions and trainings were given to each of the 11 interviewers ( 6 medical doctors, 3 nurses and 4 medical doctor students) prior to study start. The quality of data collection was ensured by regular training and monitoring of interviewers. Data were double entered and checked for completeness and plausibility.

Sociodemographic data and behavioral factors were self-reported, whereas cardio-metabolic data were checked by medical personnel using medical records (F.P.). Also, based on medical records, previous risk diseases were assessed using standard definitions for hypertension, diabetes mellitus, hypercholesterolemia, and atrial fibrillation. Body weight and body height were both measured and assessed by the questionnaire.

Active follow-up was done by phone after one, three and twelve months following the diagnosis. These contacts exclusively served to assess the occurrence of a subsequent stroke during the time period since the previous visit. Therefore, no exact event time points were given for the event "subsequent stroke". Vital status was obtained from the local population registry and date of death was assessed for deceased cases. Neither the severity nor the kind of second stroke were assessed by this follow-up.

\section{Statistical methods}

Baseline characteristics of patients are summarized with descriptive statistical methods. Continuous variables are described by means and standard deviations. Categorical data are summarized as absolute and relative frequencies.

To analyze the risk factors that might affect the time to death or subsequent stroke we applied the weighted all-cause hazard ratio, thereby taking into account the occurrence of competing events $[13,14]$. Thus, different "relevance weights" for the two competing components "death" and "subsequent stroke" were applied. For choosing the weights, we followed the recommendations for finding a weighting scheme as described by Ozga \& Rauch, which are also explained in the additional file [AdditionalFile.pdf, 14].

As a first step, we determined "death" to be the most relevant outcome, and thus a weight of " 1 " was assigned for "death". In a second step, the event "subsequent stroke" was set into relation to "death" by 
answering the purely theoretical question of "how many strokes are considered to be as harmful as one death?". The clinical consideration that "stroke" represents a severe clinical event but may be survived, thereby resulting in more "stroke events" than "deaths" within the observed time period, led us to choose a weight of 0.7 for the event "stroke". Additionally, the "disability weights" as described in the "Global Burden of Disease" study 2016 [19] supported our decision. Although the definitions of these "disability weights" are not directly comparable to our approach (i.e. weights were chosen on the basis of medical records like speech impairment, or being confined to bed or wheelchair), they also set the event "stroke" in relation to "death". Disability weights for ischemic stroke ranged from 0.019 to 0.588 with a maximal upper confidence limit of 0.744 [19].

The weighted all-cause hazard ratio was originally introduced for a two-group comparison. For our analysis we extended this effect measure to adjust for confounders [see AdditionalFile.pdf for methodological details]. In addition to the weighted all-cause hazard ratio, we also provide results for the non-weighted all-cause hazard ratio resulting from the Cox proportional hazards model and for the causespecific hazard ratios for each component separately. Along with the analysis using other weighting schemes, these later analyses are interpreted as sensitivity analyses. All hazard ratios are reported with 95\%-confidence intervals. For the weighted all-cause hazard ratio, these are estimated via bootstrap sampling with 10,000 runs. Risk diseases and risk factors evaluated at baseline were atrial fibrillation, hypertension, diabetes mellitus, hypercholesterolemia and smoking. Furthermore, all estimates were adjusted for age (continuous) and sex.

The stroke risk factors considered in this study were defined as follows (unpublished study report):

- atrial fibrillation: "persistent" and "paroxysmal"

- hypertension: values $140 / 90 \mathrm{mmHg}$ at rest at three time points

- diabetes mellitus: fasting-plasma-glucose $>125 \mathrm{mg} / \mathrm{dl}$ (>7 mmol/l) or peak blood sugar $>200 \mathrm{mg} / \mathrm{dl}$ ( $>11 \mathrm{mmol} / \mathrm{l})$ or two hours value with oral test for glucose tolerance $>200 \mathrm{mkg} / \mathrm{dl}(>11 \mathrm{mmol} / \mathrm{l})$

- smoking. consumption of either a minimum of one cigarette/day, five cigarettes/week, one pack of cigarettes/month, two cigars or two pipes/week over a period of six month or more during life

- hypercholesterolemia: fasting total serum cholesterol $200 \mathrm{mg} / \mathrm{dl}$

Since no information was available for any treatment regarding vascular risk factors and risk diseases, the therapeutic regimen could not be included as a predictor in the analysis.

As mentioned above, the exact time of an event has only been delivered for deaths but not for non-fatal strokes. In the latter cases only time intervals were presented ("interval censoring"). For unadjusted univariable analyses, some methods were proposed to account for this, such as different score tests or a parametric model [20-23]. The parametric model can be extended easily to a model adjusting for confounders. However, since we have no information about the underlying event-time distributions and a misspecification of the distribution can introduce serious bias, we decided against a parametric approach and in favor of a naive approach. If the event time is known to fall in an interval, the naive approach 
assumes that the event occurred at the upper boundary of the interval, thus at the next time point observed. To assess the robustness of this naive approach, we repeated the analyses with the event times replaced by the lower boundary of the interval and by the middle of the interval. We used the statistical software R Version 3.5.1 for all analyses [24]. The algorithm Mersenne Twister is used for random number generating needed to calculate the bootstrap confidence intervals [25].

\section{Results}

\section{Characteristics of included patients}

Baseline and further data characteristics of the 470 patients under investigation are outlined in Table 1. Physical activity and education level are only given for description of baseline characteristics but are not considered in the analysis of risk factors. Within the follow-up period of one year 23 (4.9\%) patients died and $34(7.2 \%)$ experienced a subsequent stroke. Thus, 34 event times were interval-censored. The median time-to-event (either "death" or "stroke") was 3.2 months $\left(1^{\text {st }}-3^{\text {rd }}\right.$ quantile: $\left.1.5-11.5\right)$. The median time to death was 4.1 months ( $1^{\text {st }}-3^{\text {rd }}$ quantile: 2.5 - 8.7) and the median time to subsequent stroke was 3.0 months $\left(1^{\text {st }}-3^{\text {rd }}\right.$ quantile: $\left.1.1-11.6\right)$, respectively. The remaining 413 patients were censored at 12 months plus 35 days (395 days).

\section{Table 1: Characteristics of the study population.}




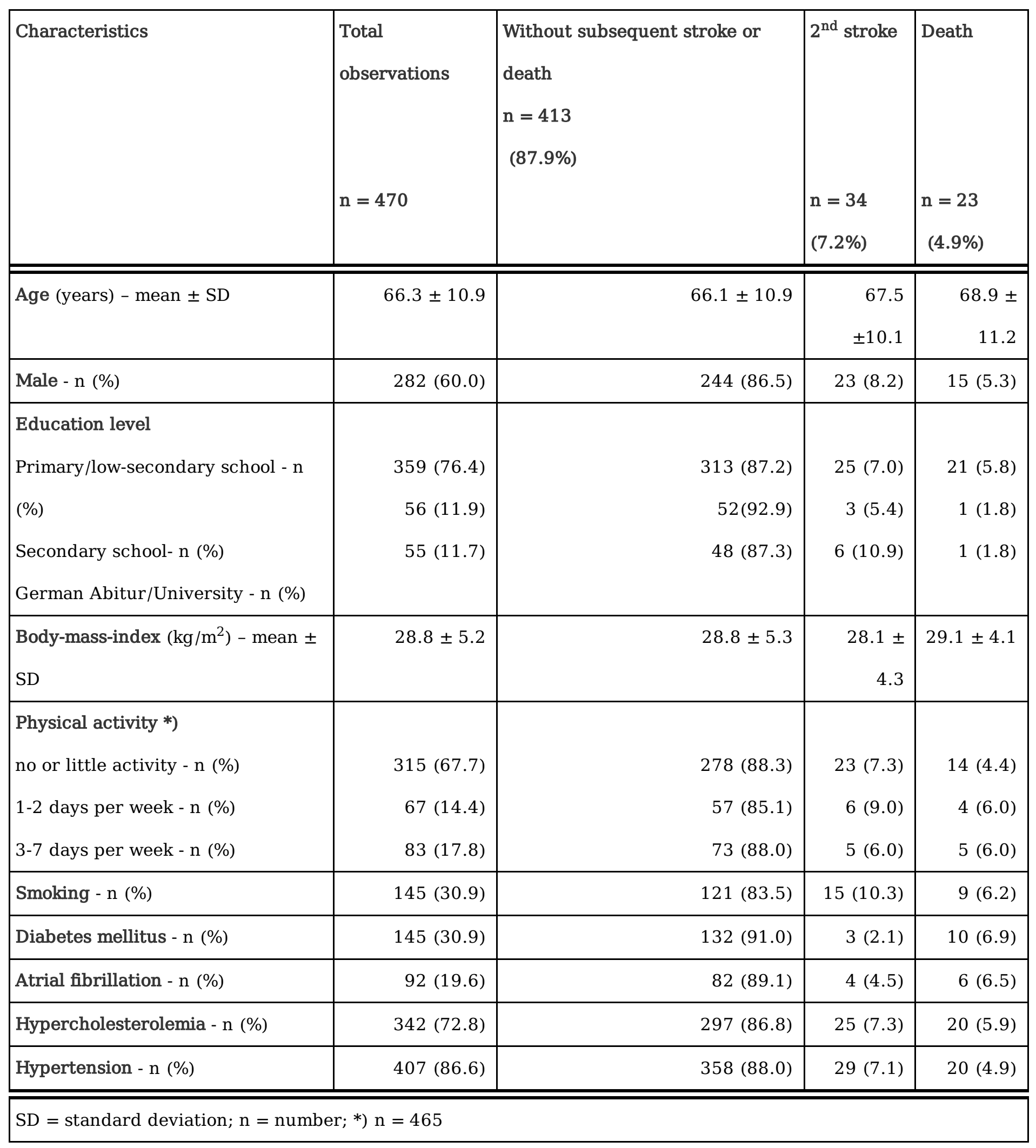

\section{Analysis of the stroke risk factors and risk diseases}

Figure 2 shows the adjusted weighted all-cause hazard ratio (HR) at 395 days after the index stroke with $95 \%$ bootstrap confidence intervals $(\mathrm{Cl})$. Figure 3 depicts the adjusted unweighted all-cause hazard ratios and the adjusted cause-specific hazard ratios for each event type separately. In each model, adjustment has been performed by including all remaining independent variables. In Figure 2 it can be seen that for 
diabetic patients the weighted average risk for a subsequent event is significantly reduced with a weighted HR of 0.39 (95\% $\mathrm{Cl} 0.20$ - 0.88) whereas none of the other known risk factors for a first ever stroke show a statistically significant association.

Furthermore, when investigating the cause-specific hazard ratios it can be seen in Figure 3 that the effect of the different event types can point into opposite directions: e.g. for diabetes mellitus, the cause-specific hazard ratio for "death" suggests a higher mortality risk $(1.75 ; 95 \%-\mathrm{Cl}=(0.74-4.12))$, but for "subsequent stroke" the event risk is virtually lower (cause-specific hazard ratio $0.23 ; 95 \%-\mathrm{Cl}=(0.07-0.75)$ ). This potentially could indicate a shift from "strokes survived" to "stroke associated with death", which would be of high clinical relevance for further clinical investigations and clarification. For the other covariates no statistically significant effects were observed.

The results for other weighting schemes are outlined in Figure 4. The differences in the estimated effects between the analyses for the unweighted all-cause effect (Figure 3) and the analysis with weight " 1 " for both event types are due to the differences in the estimated baseline hazard: For the weighted approach different cause-specific baseline hazards are assumed, whereas the model for the unweighted estimation implies the same baseline hazard for the included components. The analysis using the lower or mid interval time points for the event type "stroke" delivered similar results [see AdditionalFile.pdf].

It has to be noted that the weighted composite hazard ratio depends on the cause-specific hazards and thereby also depends on direction and magnitude of the cause-specific hazard ratios. The direction of the weighted composite effect therefore depends on these cause-specific properties and additionally on the weight choice. For larger differences between the cause-specific effects the weighting scheme has the highest impact on the weighted composite hazard ratio.

\section{Discussion}

We presented the association of known major cardiovascular risk factors and risk diseases for a first ever stroke with the combined endpoint "time to death" and "time to second stroke" during a follow-up of one year by using a newly developed method for weighted composite endpoints $[13,14]$. The presented results show that under the conditions of current treatment and secondary prevention after a first stroke, the rates of death or subsequent non-fatal strokes during the first year of follow-up are not associated anymore with the well accepted and individually ascertained cardiovascular risk factors and risk diseases. These data therefore support the hypothesis that treatment and secondary prevention measures addressing these risk factors and risk diseases are effective in the prevention of subsequent serious clinical events at least during the first year of follow-up. 
Notwithstanding, the presented data do not allow to extrapolate these observations to longer time periods, as the actual treatment options may only be effective to postpone secondary clinical events but not to completely prevent them. These events observed during the first year after a first non-fatal stroke therefore need to be evaluated in more detail to get more information on causes and deleterious mechanisms. For example, additional confounders might influence the association seen for diabetic patients, especially with respect to life style and comorbidities. Furthermore, the degree of organ damage also influences the disease progress but could not be explicitly incorporated in the model due to missing information. The time point of starting therapeutic interventions with regard to primary and secondary prevention of stroke also will affect the occurrence of subsequent events during follow-up. Thereby, it is a limitation of this study that the specific treatments including medications could not be included into this evaluation.

The study has some further limitations: The sample size and thus the number of events is small. The mortality in the study group is low due to the selection of "cases" within the underlying case-control study, in which strokes with severe impairment were excluded. However, similar stroke rates were found in other studies $[3,8,26]$. The low event number also results in a limited accuracy of the estimates as expressed by wide confidence intervals. Furthermore, secondary stroke records were based on the patients' information, which was not verified by medical supervision. The severity of secondary strokes has not been assessed which would have allowed to use individual weights. Additionally, we did not incorporate a possible change of patients' conditions over time, e.g. if patients stopped smoking. Finally, there was no documentation of the causes of death.

As a methodological result, our analysis emphasizes the importance to take all event types simultaneously into consideration to derive meaningful interpretations. Thereby, the different clinical relevance of competing event types should be taken into account, which can be done with the weighted all-cause hazard ratio yielding a weighted average effect. This methodology considerably strengthens the basis of associations measured in clinical studies and registries due to the consideration of all event times and the length of an individual being at risk for any event. Thus, a disease progress can be more adequately described. Serving as a model for this biometrical approach the present study clearly shows that the associations of well-known risk factors and risk diseases for the occurrence of a first stroke do not apply anymore during the period of secondary prevention. By this new methodologic approach our study differs from other studies, which exclusively examined either the risk for a recurrent stroke or the risk for death [7-9]. However, choosing the appropriate weights remains to be challenging and should be further discussed in detail by medical and statistical experts.

\section{Conclusion}

In summary, we, for the first time, extended the weighted all-cause hazard ratio to adjust for confounders. Thus, it is now generally possible to assess several risk factors by a time-to-event analysis for composite endpoints, thereby incorporating competing endpoints of different clinical relevance. By this way, disease progress can more adequately be described, which is of high relevance for an individualized and clinically 
successful treatment taking into account the individual risk profiles and comorbidities and thereby allowing a highly individualized therapy as precondition for a successful secondary prevention.

Against this background, our results suggest that most commonly reported risk factors and risk diseases for a first stroke cannot serve as predictors for subsequent stroke events or death anymore. The clinical relevance of our results is limited due to the small number of events and should be verified by testing the presented methodology on the basis of larger populations.

\section{List Of Abbreviations}

Cl: confidence interval; HR: hazard ratio

\section{Declarations}

Ethics approval and consent to participate. The study was approved by the ethics committee of the Landesärztekammer Rheinland-Pfalz (837.333.05(4991)).

Consent for publication. Not applicable.

Availability of data and materials. The datasets used and analyzed during the current study are available from the corresponding author on reasonable request.

Competing interests. The authors declare that they have no competing interests.

Funding. This work was supported by the German Research Foundation [Grant RA 2347/1-2]. HB was partly funded by Stiftung Charité. The German Research Foundation and Stiftung Charité had no influence on any of the research, i.e. study design, analysis, interpretation, or writing, done in this article.

Authors' contributions. AO implemented the statistical analysis, produced the results and wrote the first draft of the manuscript. GR and HB contributed to all parts of the manuscript. All authors read and approved the final manuscript. BR, FP, CU, and, AG were contributors in writing the manuscript

Acknowledgements. We thank Annette Aigner for helping with the data management and Mrs. Caroline Wolff for her professional advice with regards to the English language.

\section{References}


1. Lozano R, Naghavi M, Lim S, Shibuya K, Aboyans V, Abraham J, et al. Global and regional mortality from 235 causes of death for 20 age groups in 1990 and 2010: a systematic analysis for the Global Burden of Disease Study 2010. Lancet. 2012; 380: 2095-2128.

2. Murray CJ, Vos T, Lozano R, Naghavi M, Flaxman AD, Michaud C, et al. Disability-adjusted life years (DALYs) for 291 diseases and injuries in 21 regions, 1990-2010: a systematic analysis for the Global Burden of Disease Study 2010. Lancet. 2012; 380: 2197-2223.

3. Hankey GJ, Jamrozik K, Broadhurst RJ, Forbes S, Burvill PW, Anderson CS, et al. Five-year survival after first-ever stroke and related prognostic factors in the Perth Community Stroke Study. Stroke. 2000; 31:2080-2086.

4. Brønnum-Hansen, H; Davidsen, M; Thorvaldsen, P; Danish MONICA Study Group. Long-term survival and causes of death after stroke. Stroke. 2001;32:2131-2136.

5. Becher H, Grau A, Steindorf K, Buggle F, Hacke W. Previous infection and other risk factors for acute cerebrovascular ischaemia: attributable risks and the characterisation of high risk groups. J Epidemiol Biostat. 2000; 5:277-283.

6. Gorelick PB. Stroke prevention therapy beyond antithrombotics: unifying mechanisms in ischemic stroke pathogenesis and implications for therapy: an invited review. Stroke. 2002; 33:862-875.

7. Fu GR, Yuan W, Du W, Yang Z, Fu N, Zheng H, et al. Risk Factors Associated with Recurrent Strokes in Young and Elderly Patients: A Hospital-based Study. Int J Gerontol. 2015; 9:63-66.

8. Wangqin $R$, Wang $X$, Wang $Y$, Xian $Y$, Zhao X, Liu L, et al. Risk factors associated with 90-day recurrent stroke in patients on dual antiplatelet therapy for minor stroke or high-risk TIA: a subgroup analysis of the CHANCE trial. Stroke Vasc Neurol. 2017; 2:176-183.

9. Leoo T, Lindgren A, Petersson J, von Arbin M. Risk factors and treatment at recurrent stroke onset: results from the Recurrent Stroke Quality and Epidemiology (RESQUE) Study.

Cerebrovasc Dis. 2008; 25:254-260.

10. Zheng S, Yao B. Impact of risk factors for recurrence after the first ischemic stroke in adults: A systematic review and meta-analysis. J Clin Neurosci. 2019; 60:24-30.

11. Feigin VL, Norrving B, Mensah GA. Global Burden of Stroke. Semin Neurol. 2018; 38: 208-211.

12. Oza R, Rundell K, Garcellano M. Recurrent Ischemic Stroke: Strategies for Prevention. Am Fam Physician. 2017; 96: 436-440.

13. Rauch G, Kunzmann K, Kieser M, Wegscheider K, König J, Eulenburg C. A weighted combined effect measure for the analysis of a composite time-to-first-event endpoint with components of different clinical relevance. Stat Med. 2018; 37:749-767.

14. Ozga AK, Rauch G. Introducing a new estimator and test for the weighted all-cause hazard ratio. BMC Med Res Methodol. 2019; 19:1-16.

15. Becher H, Palm F, Aigner A, Safer A, Urbanek C, Buggle F, et al. Socioeconomic Conditions in Childhood, Adolescence, and Adulthood and the Risk of Ischemic Stroke. Stroke. 2016; 47:173179.

16. Palm F, Urbanek C, Rose S, Buggle F, Bode B, Hennerici MG, et al. Stroke Incidence and Survival in Ludwigshafen am Rhein, Germany: the Ludwigshafen Stroke Study (LuSSt). Stroke. 2010; 41:1865-1870.

17. Truelsen T, Begg S, Methers C. The global burden of cerebrovascular disease.

https://www.who.int/healthinfo/statistics/bod_cerebrovasculardiseasestroke.pdf. Accessed 8 
March 2019

18. Vuksanović M, Safer A, Palm F, Stieglbauer G, Grau A, Becher H. Validity of self-reported BMI in older adults and an adjustment model. J Public Health. 2014; 22:257-263.

19. GBD 2016 Disease and Injury Incidence and Prevalence Collaborators. Global, regional, and national incidence, prevalence, and years lived with disability for 328 diseases and injuries for 195 countries, 1990-2016: a systematic analysis for the Global Burden of Disease Study 2016. Lancet. 2017; 390:1211-1259.

20. Fay MP. Comparing Several Score Tests for Interval Censored Data. Stat Med. 1999; 18:273285.

21. Finkelstein DM. A Proportional Hazards Model for Interval-Censored Failure Time Data. Biometrics. 1986; 42:845-854.

22. Finkelstein MD, Wolfe RA. A Semiparametric Model for Regression Analysis of IntervalCensored Failure Time Data. Biometrics. 1985; 41:933-945.

23. Lindsey JC, Ryan LM. Tutorial in biostatistics methods for interval-censored data. Stat Med. 1998; 17:219-238.

24. R Core Team. R: A Language and Environment for Statistical Computing (Version 3.5.1). http://www.R-project.org/. Accessed March 2019

25. Matsumoto M, Nishimura T. Mersenne twister. A 623-dimensionally equidistributed uniform pseudorandom number generator. ACM Trans Model Comput Simul. 1998; 8:3-30.

26. Amerenco P, Lavallée PC, Labreuche J, Albers GW, Bornstein NM, Canhão P, et al. One-Year Risk of Stroke after Transient Ischemic Attack or Minor Stroke. N Engl J Med. 2016; 374:1533-1542.

\section{Additional File Information}

Additional file. The additional file [AdditionalFile.pdf] contains the description of the "Adjusted weighted all-cause hazard ratio" as well as general considerations for choosing the weights, an illustration of correlation between independent variables, and further results for the estimated weighted hazard ratio using the lower or mid interval time points for the event "stroke" and the weights 0.7 for "stroke" and 1 for "death".

\section{Figures}




\section{$1^{\text {st }}$ study:}

"The Ludwigshafen Stroke Study"

- population-based registry (2006-2017)

- $\mathrm{n}=1231$ cases with stroke or TIA

- $\mathrm{n}=725$ first ever strokes

$2^{\text {nd }}$ study:

"Socioeconomic Conditions in Childhood, Adolescence, and Adulthood and the Risk of Ischemic Stroke"

- case-control study

- $\mathrm{n}=470$ cases with first ever stroke from $1^{\text {st }}$ study (2007-2012)

- Matched controls were taken from the general population of the Ludwigshafen area.

\section{Present study}

- $\mathrm{n}=470$ cases of the $2^{\text {nd }}$ study with follow-up information taken from $1^{\text {st }}$ study

Figure 1

Flow chart describing basis of study sample. 
Diabetes mellitus

Hypercholesterolemia

Hypertension

Smoking

Confounders:

Age

Male sex
Atrial fibrillation

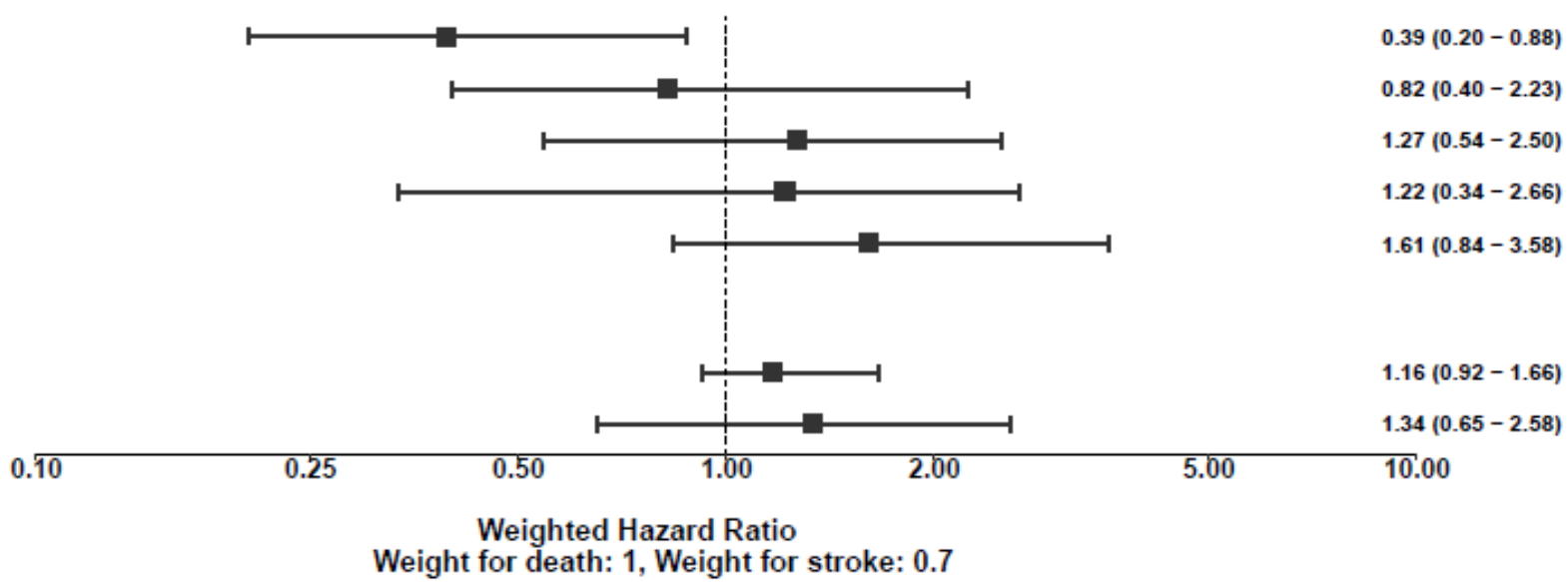

Figure 2

Adjusted weighted hazard ratios with weights " 1 " for death and "0.7" for stroke. All effects are adjusted for all other independent variables. Confidence intervals were produced via bootstrap sampling. For age the hazard ratio refers to a ten year`s difference.

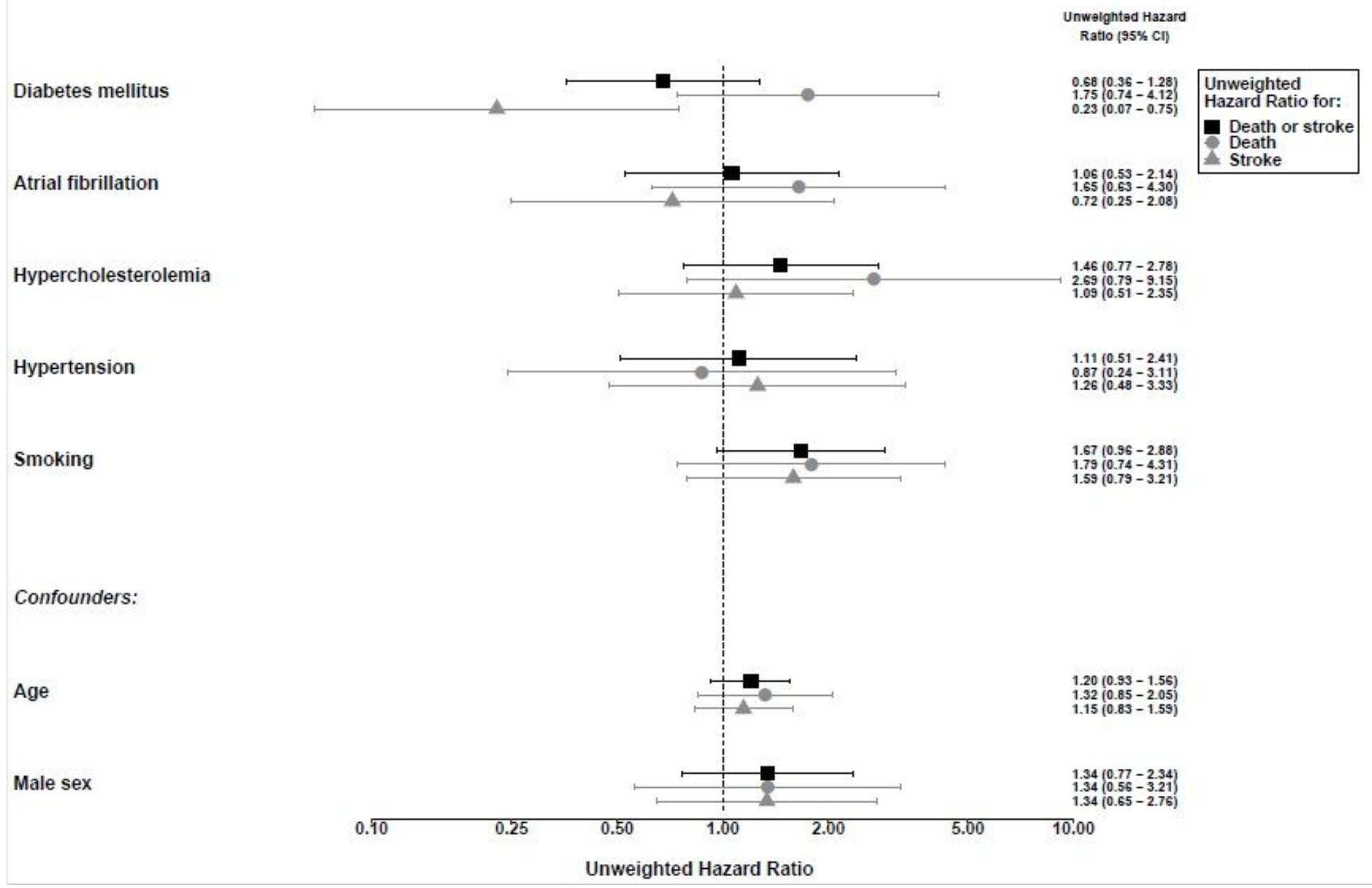


Adjusted unweighted all-cause hazard ratio and cause-specific hazard ratios for sensitivity analysis. All effects are adjusted for all other independent variables. Confidence intervals were produced via bootstrap sampling. For age the hazard ratio is for a ten year's difference.

Diabetes mellitus

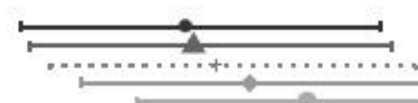

Weighted Hazard

Ratio $(95 \% \mathrm{Cl})$

$0.35\left[\begin{array}{l}0.18-0.76 \\ 0.36\end{array}\right.$

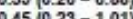

$0.570 .29-1.30$

$0.93(0.45-2.31)$

Weight for death: 1

Weight for stroke:

$* 1$

요 0.9

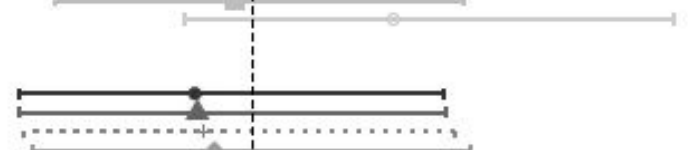

$079039-213$

$0.80(0.39-2.15)$

$0.820 .40-2.23)$

$0.8660 .41-2.38$,

$0.93(0.44-2.74$
$1.15(0.52-3.98$

40.7

+0.5
0.3

Atrial fibrillation

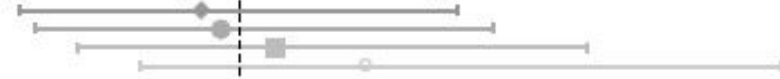

$1.65(0.67-8.68)$

0.1

Hypercholesterolemia

Hypertension

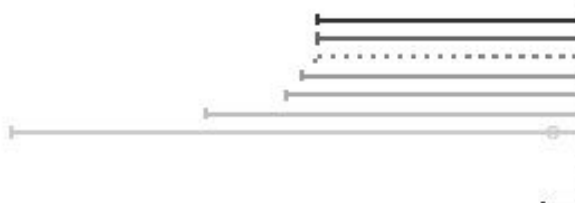

Smoking

Confounders:

Age

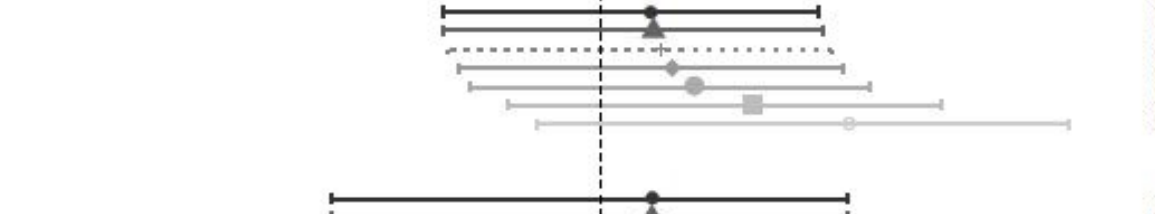

$122(0.53-2.39)$

$1.27(0.54-2.50)$

$5(0.59-2.93)$

$2.69(0.78-6.47)$

$123 \mid \begin{array}{ll}123 \\ 1.34-2.67 \\ 0.34-2.66\end{array}$

$1.220 .34-2.66$

$12000.32-2.66$

$1.08(0.22-2.58)$
$0.87(0.10-2.32)$

$1.61(0.84-3.51)$

$1.61(0.84-3.53)$

$1.61[0.84-3.58 \mid$

$1.63(0.81-3.82)$

$1.79(0.78-5.58)$

$1.16(0.92-1.65)$

$1.16[0.92-1.65)$

$11702-1.68$

$1.180 .93-1.70)$

$1.32(0.84-1.92)$

Male sex

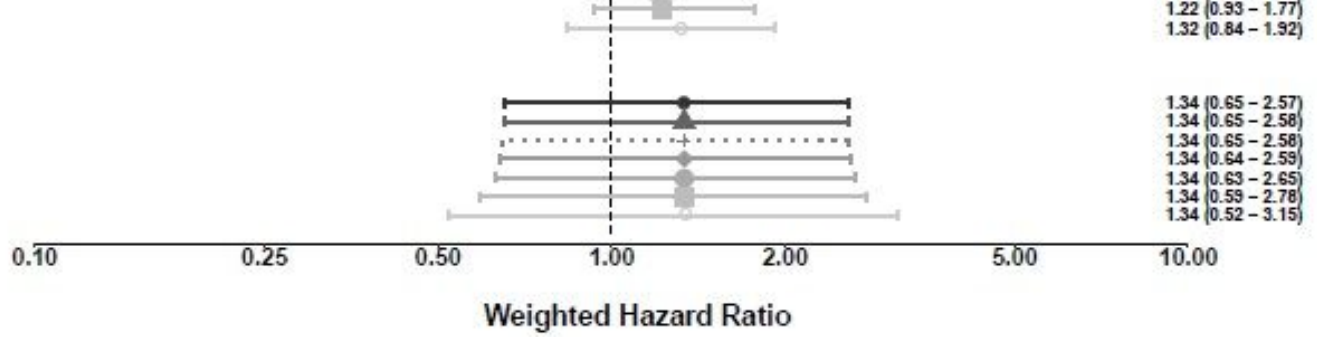

Figure 4

Adjusted weighted hazard ratios with weight " 1 " for death and weights for stroke ranging between " 0 " and "1" for sensitivity analysis. All effects are adjusted for all other independent variables. Confidence intervals were calculated via bootstrap sampling. For age the hazard ratio is for a ten year's difference.

\section{Supplementary Files}

This is a list of supplementary files associated with this preprint. Click to download. 
- AdditionalFile.pdf

Page 16/16 